\title{
ANALYSIS OF THE DYNAMIC BEHAVIOUR OF THE CIRCUIT OF A COMMON RAIL DIRECT INJECTION SYSTEM
}

\author{
Anne-gaël FAVENNEC ${ }^{*}$, Pascale MINIER ${ }^{*}$, Michel LEBRUN ${ }^{* *}$ \\ * Research Department, RENAULT \\ 1 , avenue du golf - 78288 Guyancourt cedex, France \\ (anne-gael.favennec@renault.com - pascale.minier@renault.com) \\ ** Department of Mechanics, Université Claude Bernard LYON I, France
}

\begin{abstract}
If it is recognised these days that simulation plays a central role in the design process, this should go hand-in-hand with methodology. We intend to underline these metholodological aspects by developing the modelling and simulation of a direct injection system known as "Common Rail". This modelling is developed on the basis of an elementary models' library facilitating the use of a "polymorphic" approach, that allows to analyse both the stucture of the developed models and the parameters influences.
\end{abstract}

\section{KEY WORDS}

Direct Injection, Linear and non linear Analysis

\section{INTRODUCTION}

This paper deals with Common Rail type injection system's modelling, in oder to analyse its behaviour and propose a methodology to design the hydraulic network. Models of each component which make up the injection system are described. It rests on a breakdown of the engine network into volumes and hydraulic lines modelled by finite differences. A linear analysis around an operating point is then presented from which the eigen values, the modal forms and bode diagrams associated with different transfer functions of the circuit are calculated. The consequent understanding of the system allows optimisation of the network design, and the choice of hypothesis level just sufficient to obtain temporal simulations of the complete injection system with reasonable calculation time span and good analysis and understanding of the system behaviour

\section{COMPONENTS MODELLING}

The injection system is intended to inject, into each cylinder of the engine, and for each injection, quantities of fuel of several $\mathrm{mm}^{3}$ to several tens $\mathrm{mm}^{3}$ according to the operating conditions. The length of an injection varies from one fraction of milli-second (in preinjection phase) to several milli-seconds. This device is made-up of a feed pump, a high-pressure pump ( $\max$ 1350 bar), a pressure regulator piloted by PWM, electrically controlled injectors, flow limitors and a distribution network linking these parts as shown in figure 1. 


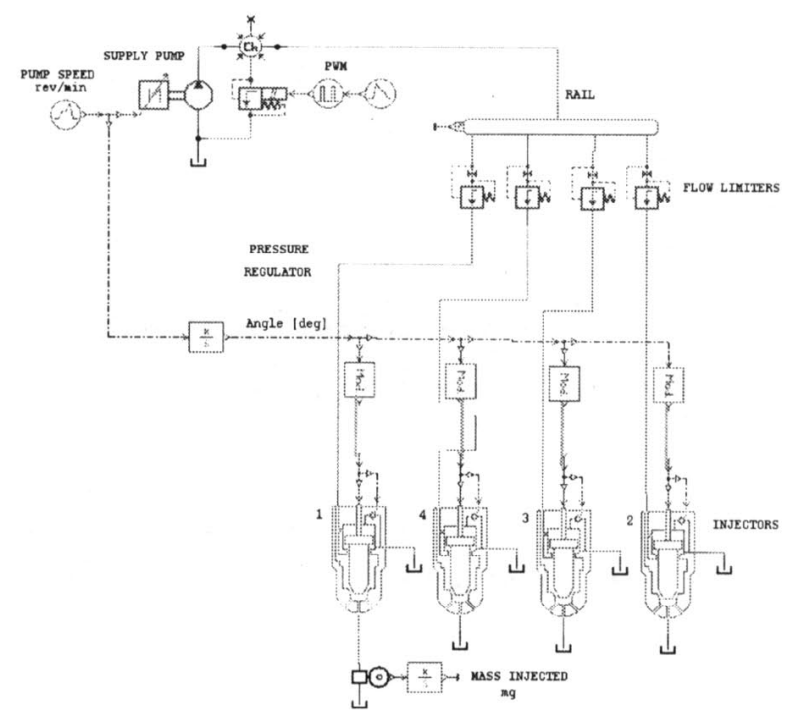

Figure 1 Common rail system

The following paragraph presents the modelling of components which make up the injection system. They are developed using the elementary hydraulic components library (AME Basic ELement [2]) of AMESim software [1].

\section{High pressure pump}

A representation of the high pressure pump is shown in figure 2. The model concerns a pump with 3 radial pistons with check valves. The feed pump is modelled by a constant source of pressure.

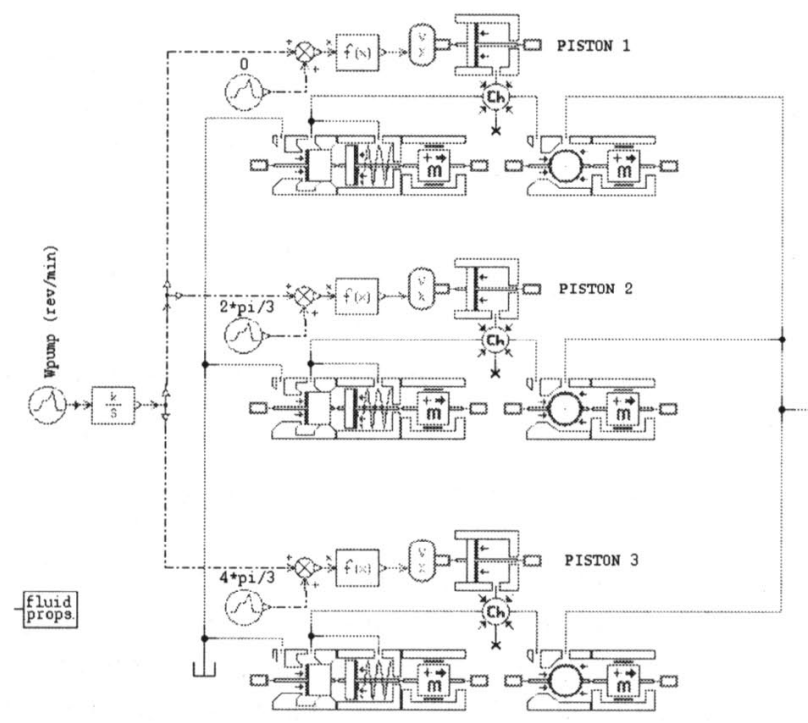

Figure 2 high pressure pump

The model is tested by connecting it to an orifice adjusted to give 1000 bar at the pump outlet. the average flow rate in the orifice, and the evolution of the pressure into the volume are determined. With the figure 3, result of a F.F.T. (Fast Fourier Transformation) applied to the pressure signal, we get the $f_{i}$ frequencies representing the fundamental and the harmonics linked to the pump kinematic $\left(f_{i}=2 i N \omega\right)$. Moreover, we find a first frequency $N \omega$, . This phenomenon is due to the compressibility effect in pump dead volumes, and to the kinematic of check valves.

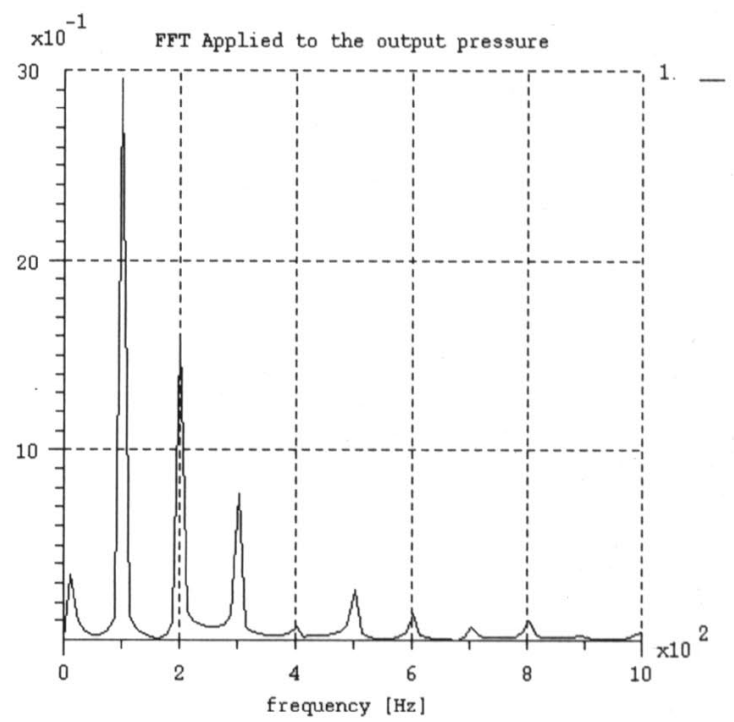

Figure 3 Fast Fourier Transform (FFT) of the output pressure [bar]

\section{Pressure regulator}

The regulator is designed to give a constant average pressure in the network that is relatively independent of the consumption flow through the injectors. This pressure is controlled by an electromechanical unit. The model is given in figure 4 . The electromagnetic force is given by a table with 2 dimensions, the current $i$ and the position $x$ of the ball. A P.W.M. tension (Pulse Width Modulator) is created by a signal source.

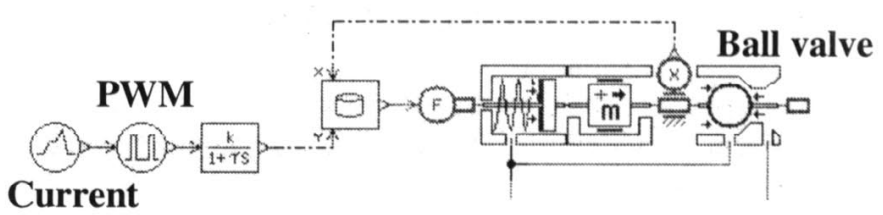

Figure 4 pressure regulator model

\section{Flow limitor}

The Flow limiter is introduced into the network as a safety device to close the line connected to the injector if there is a leakage. A model is shown in figure 5. This component is sized with the injector, so that in normal operation it does not close. It does, however, introduce a certain damping effect into the network. 


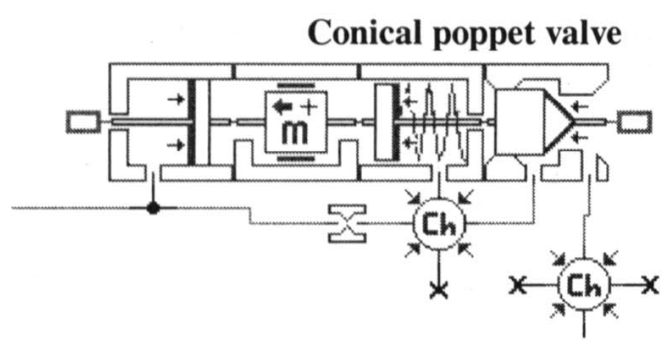

Figure 5 flow limiter model

\section{Injector}

A model of a Bosch Common Rail injector is presented in Figure 6.

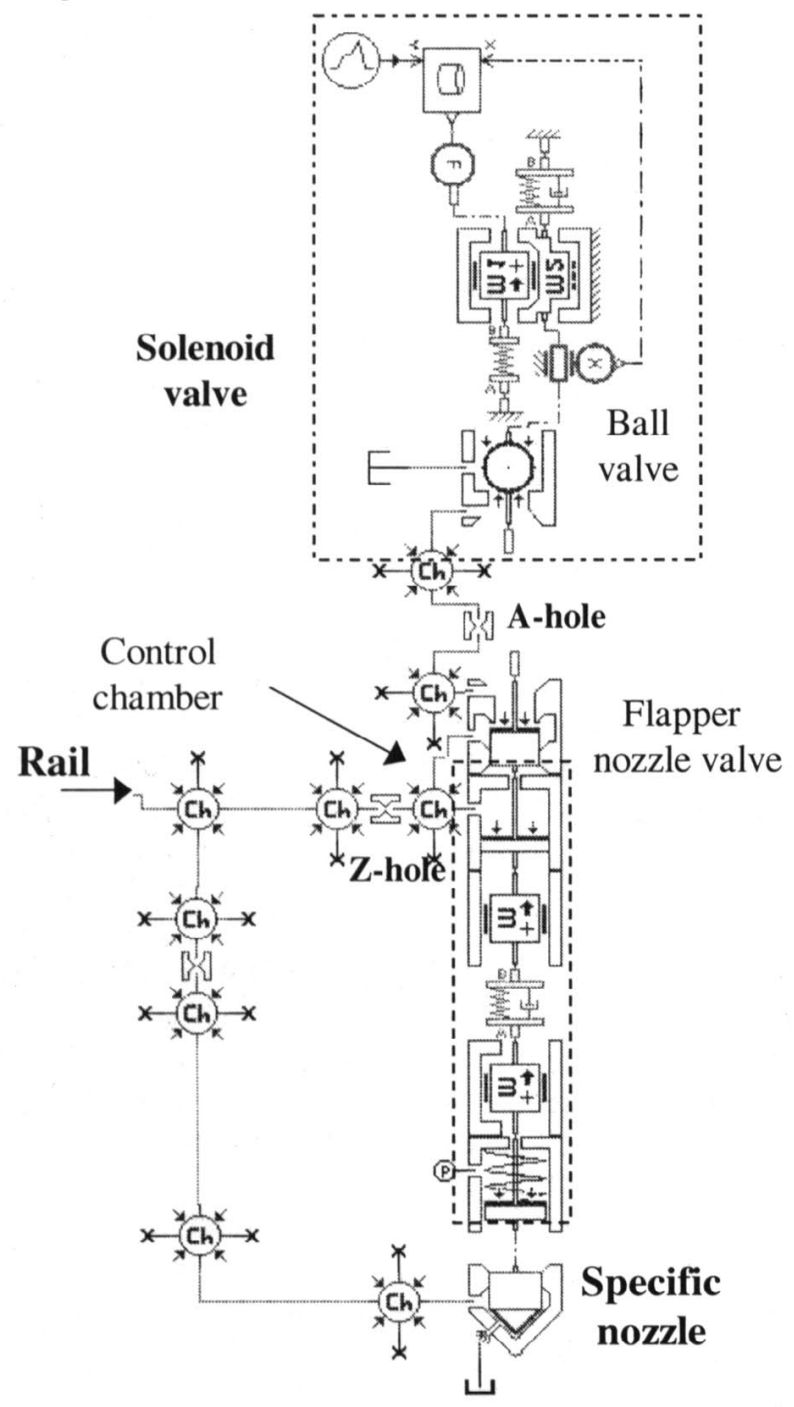

Figure 6 AMEBel injector Model

The model takes into account the elasticity of the needle. A global modelling of the injector nozzle has been choose [3]. Indeed, a global flow coefficient $C_{q}$ has been determined with experimental data, as a function of the cavitation number $k$ and the flow number $\lambda$. The magnetic force is given by a table with 2 dimensions, as for the pressure regulator.

\section{Fluid properties}

Models of fluid properties take into account the following phenomena:

- variable density $\rho$, function of the pressure and the temperature

- the variable viscosity $v$, function of the pressure and the temperature

- the consistency between density and compressibility in order to ensure mass conservation $\beta=\rho \frac{\partial \rho}{\partial \rho}$

The expansion of the pipe wall with pressure is taken into account by an effective bulk modulus. Figures 7 and 8 show the evolution of the characteristics of the test bench used fluid according to two pressure ranges.

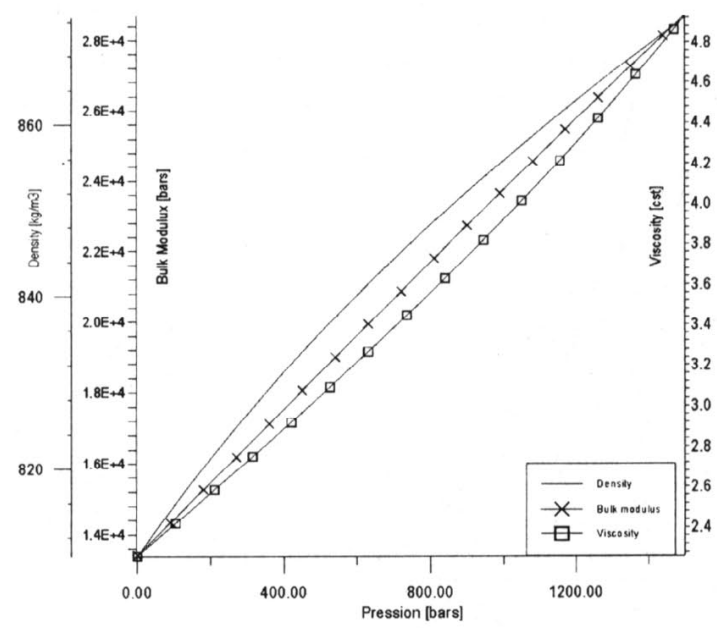

Figure 7 Fluid properties between 0 and 1500 bar

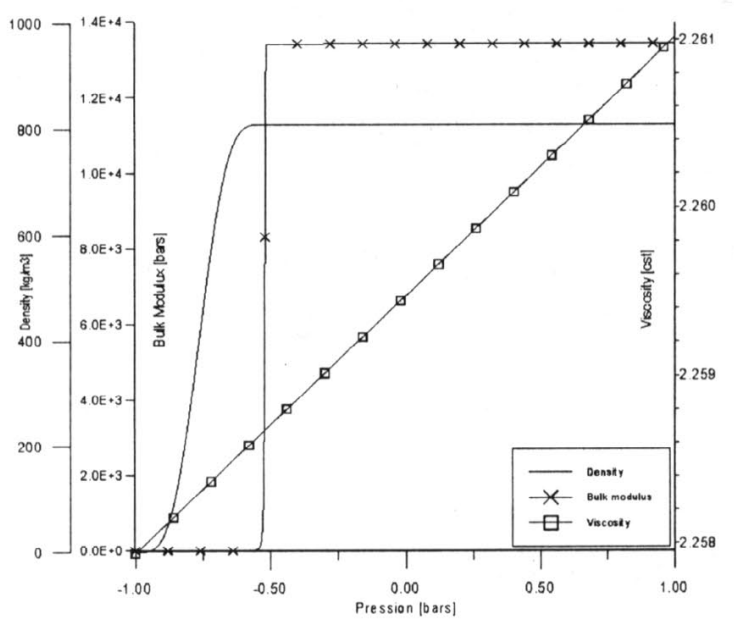

Figure 8 Fluid properties between -1 and 1 bar

Between -1 and 1 bar, we show the evolution of the properties in aeration and/or cavitation phase (local pressure inferior to the pressure of saturating vapor). Between 0 and 1500 bar, we demonstrate the significant evolution of the fluid properties. 


\section{HYDRAULIC NETWORK}

The analysis of the dynamic behavior of a hydraulic line is relatively complex. The behavior of a line can be dominated by :

- compressibility effect,

- compressibility and dissipative effects,

- wave effect where the inertia compressibility and dissipative effects are significantly coupled [1].

For a hydraulic network, the interaction between lines of different characteristics (length, diameter, stiffness) induce a behavior which is difficult to analyse. Due to these interactions, the choice of the line models is not easy. If we take a complex line model at each part of the circuit, the size of the state equation will be enormous. The simulation time will be unacceptable. If the model is too simple the results will be poor. From these considerations it appears that time domain simulations are not well-adapted to analyze the network and choose the best models. A better alternative is a linear analysis approach.

\section{Linear anlysis}

The linear model shows the intrinsic properties of the system, independent of the inputs. With tools as transfer functions or modal shape analysis, it is simpler to analyse than a non-linear model. It is particularly useful when the excitation of the circuit is periodic as ripple of the flow rate from the pump, opening and closing of the injectors at a certain frequency... In this case, the analysis of the transfer function and the spectrum of the entries permits to understand the time response of the system and then help in choosing the type of line model of the different parts of the network. The calculation of the time response of the non-linear model can be minimized by this approach.

We have a non-linear model with the classical form :

$$
\left\{\begin{array} { l } 
{ \dot { x } = f ( x , u , t ) } \\
{ y = g ( x , u , t ) }
\end{array} \text { or } \quad \left\{\begin{array}{l}
0=f(\dot{x}, x, u, t) \\
0=g(y, x, u, t)
\end{array}\right.\right.
$$

We are able to calculate the linear tangent solution.

$$
\left\{\begin{array}{l}
\dot{x}^{*}=A \cdot x^{*}+B \cdot u^{*} \\
y^{*}=C \cdot x^{*}+D \cdot u^{*}
\end{array}\right.
$$

This linear state equation is available at the equilibrium point (with $\dot{\boldsymbol{x}}=0$ and $u=$ constant). If we consider the T matrix such as $\Lambda=\mathbf{T}^{\mathbf{- 1}} \mathbf{A T}$ is diagonal, the terms of the diagonal of $\Lambda$ are the eigenvalues $\lambda_{i}$ and $\mathrm{T}$ matrix represents the eigenvectors.
Now, we can consider the initial system represented in the modal basis such as :

$$
\left\{\begin{array}{l}
\dot{z}=T^{-1} A T z+T^{-1} B u \\
y=C T z
\end{array}\right.
$$

The solution of this system is :

$$
z_{i}=e^{\lambda_{i} t} z_{i}(0)+\int_{0}^{t} e^{\lambda_{i}(t-r)} V_{i}(r) d r
$$

If we consider z $(0)=0$ and $V_{i}=\delta(0)$ (Dirac function), the solution becomes :

$$
\left\{\begin{array}{l}
z_{i}=e^{\lambda_{i} t} \\
\underline{y}=C T \underline{z}(t)
\end{array}\right.
$$

where $\underline{y}$ is a modal shape.

With this result it is interesting to consider two cases :

- Case $1: \lambda_{\mathrm{i}}$ are real or complex conjugate pairs with a non-zero real part. In this case, the modal shape evolves with time.

- Case $2: \lambda_{\mathrm{i}}$ are purely imaginary. The system is conservativ (no dissipation), the mode shape has an oscillation form.

The interpretation of the first case is not easy without animation (except with a simple system). The second case is easy to represent. Our attention is concentrated on the second case. In many hydraulic circuits, damping is very low and the situation approximates to case 2 .

\section{Analysis of the eigenvalues and modal shapes}

From this calculation, if we adopt $y$ such as that $y$ is a velocity vector at different points of the circuit, we are able to calculate the modal shapes corresponding to each eigenvalue. Lets consider the hydraulic network of the figure 9.

We just took into account volumes and lines of the network. We gave a number to each line, and an arbitrary positive direction. At the beginning of this work, the rail is modelled as five lines : we take into account inertia of fluid in each lump. The line between the pump and rail is lumped into 6 nodes. The other lines are modelled with only one inertia. The model has got 140 state variables, and 38 observer variables (velocity of each inertia). After the linearisation of our network with AMESim software, we have calculated the eigenvalues and their modal shapes. For the modal shape, we use velocity instead of flow, because the velocity is more representative of the wave phenonenun 


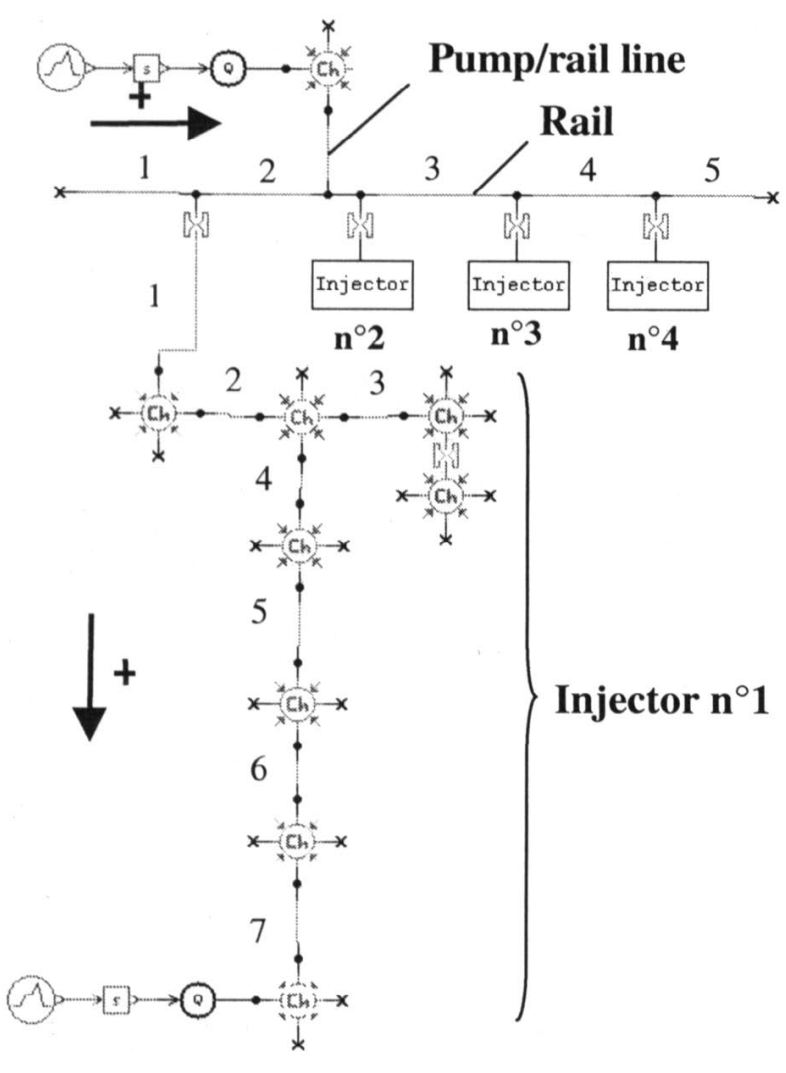

Figure 9 Common rail hydraulic network

Figure 11 shows the modal shape for the fourth eigenvalue : $695 \mathrm{HZ}$, with a rail diameter of $11,5 \mathrm{~mm}$. This mode is identified as issued from the injectors. Indeed, when observing velocities, it appears major flows in these injectors lines. Likewise, the other modal shapes reflect excitation of each lump. In oder to have a source of pressure in front of the injectors, the rail must decouple the effect of the pump on the injectors, of one injector on the others during an injection. So we plotted the Bode diagram of the transfer function between the pressure $P$ in the line between the rail and the third injector, and the integrated pump flow $Q$, for different rail diameters, on figure 10 . The static gain is given by:

$$
\frac{P}{\int Q}=\frac{\beta}{V}
$$

where $\beta$ is the compressibility of the fluid, and $V$ the total volum of the network.

There are 3 resonance peaks at 695, 1114 and $2060 \mathrm{~Hz}$, for the $11.5 \mathrm{~mm}$ rail diameter. The second frequence is identified like the first mode of the line between the pump and the rail, the third frequence represents a movement of fluid only in the rail. The diameter increase ensures a better decoupling between the pump and the injectors, since the gain is greatly decreased on the first two resonances (min $10 \mathrm{~dB}$ ).

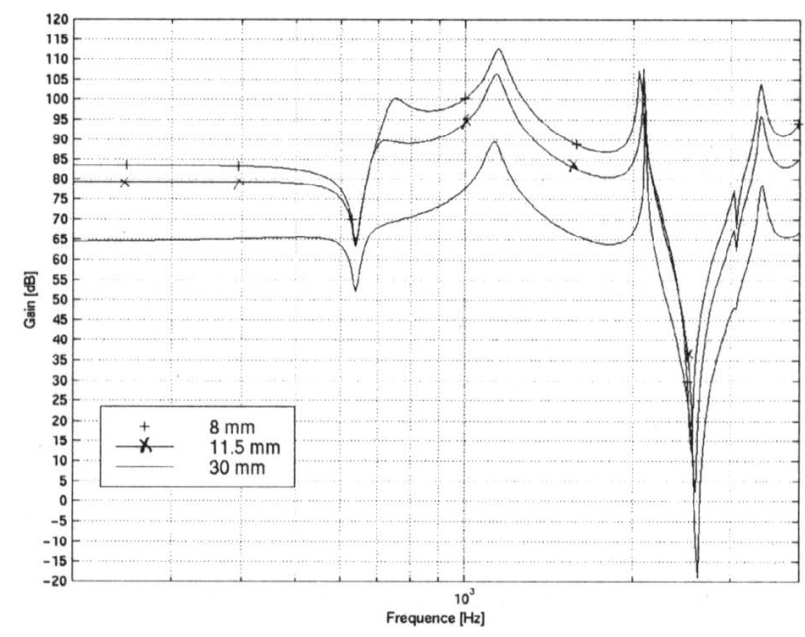

Figure 10 Bode Diagram of the ratio of injector pressure and integrated pump flow

The optimum rail diameter can then be choose with the injection system specifications. Futhermore, the pump spectrum (figure 3 ) does not excite the frequency above $1000 \mathrm{~Hz}$. Thus the frequence of $2060 \mathrm{~Hz}$ will never be excited by the pump. Likewise, the analysis of the transfer function between the pressure on the railinjector line and the derived of the injector flow rate, and, the analysis of the spectrum of this flow rate (for a $11.5 \mathrm{~mm}$ rail diameter) demontrates that an injector cannot excite frequencies over $1500 \mathrm{~Hz}$. Thus at the temporal level, we only need to correctly model frequencies inferior to $1500 \mathrm{~Hz}$. Moreover, the velocity in the rail is lower than those of the other lines. For each eigenvalue, the velocity is null or very low, compare to the other (modal shapes). So we don't have to take into account any wave in the rail. We just have to considere it as a compressible volume, with this diameter of $11,5 \mathrm{~mm}$, and this length. We proceed in a same way with the other lines and can thus choose the line models to be retained for temporal simulation.

Simulation of the complete system

Now, we are able to simulate the complete system. We present the results of simulation on figure 12 . The transient behaviour fits well, although the range is not always similar to the measurements. learning process and the design process. A good methodology can be applied to analyze and understand the studied system.

\section{CONCLUSION}

This technical note highlights two important points. We have modelled a complex circuit such as an injection system. the Method is adapted to help the learning and the design process. A good methodology can be applied to analyze and understand the system studied. 
NOMENCLATURE

$\begin{array}{ll}N & \text { Number of pump pistons } \\ \omega & \text { Shaft speed } \\ f & \text { Frequence } \\ P & \text { Pressure } \\ Q & \text { Flow rate } \\ V & \text { Volume } \\ \rho & \text { Density } \\ \beta & \text { Bulk modulus } \\ \nu & \text { Viscosity }\end{array}$

$\boldsymbol{K}=\frac{\boldsymbol{P}_{\text {up }}-\boldsymbol{P}_{\text {down }}}{\boldsymbol{P}_{\text {down }}} \quad$ Cavitation number

$\lambda=\frac{\boldsymbol{d}_{\boldsymbol{h}}}{v} \sqrt{\frac{2}{\rho}\left(\boldsymbol{P}_{u p}-\boldsymbol{P}_{\text {down }}\right)}$ Flow number

\section{REFERENCES}

[1] IMAGINE - AMESim manual

[2] IMAGINE - AMEBel manual

[3] FAVENNEC, LEBRUN - Models for Injector nozzle - The Sixth Scandinavian International Conference on Fluid Power- May 26,28 1999

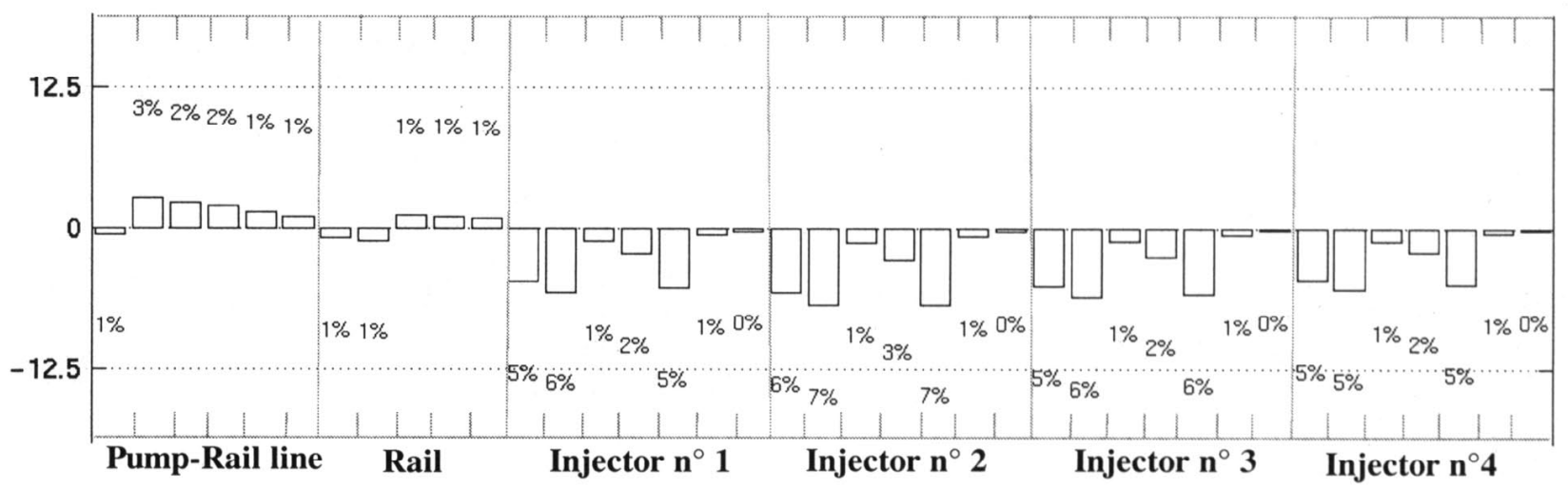

Figure 11 Modal shape of the fourth eigenvalue
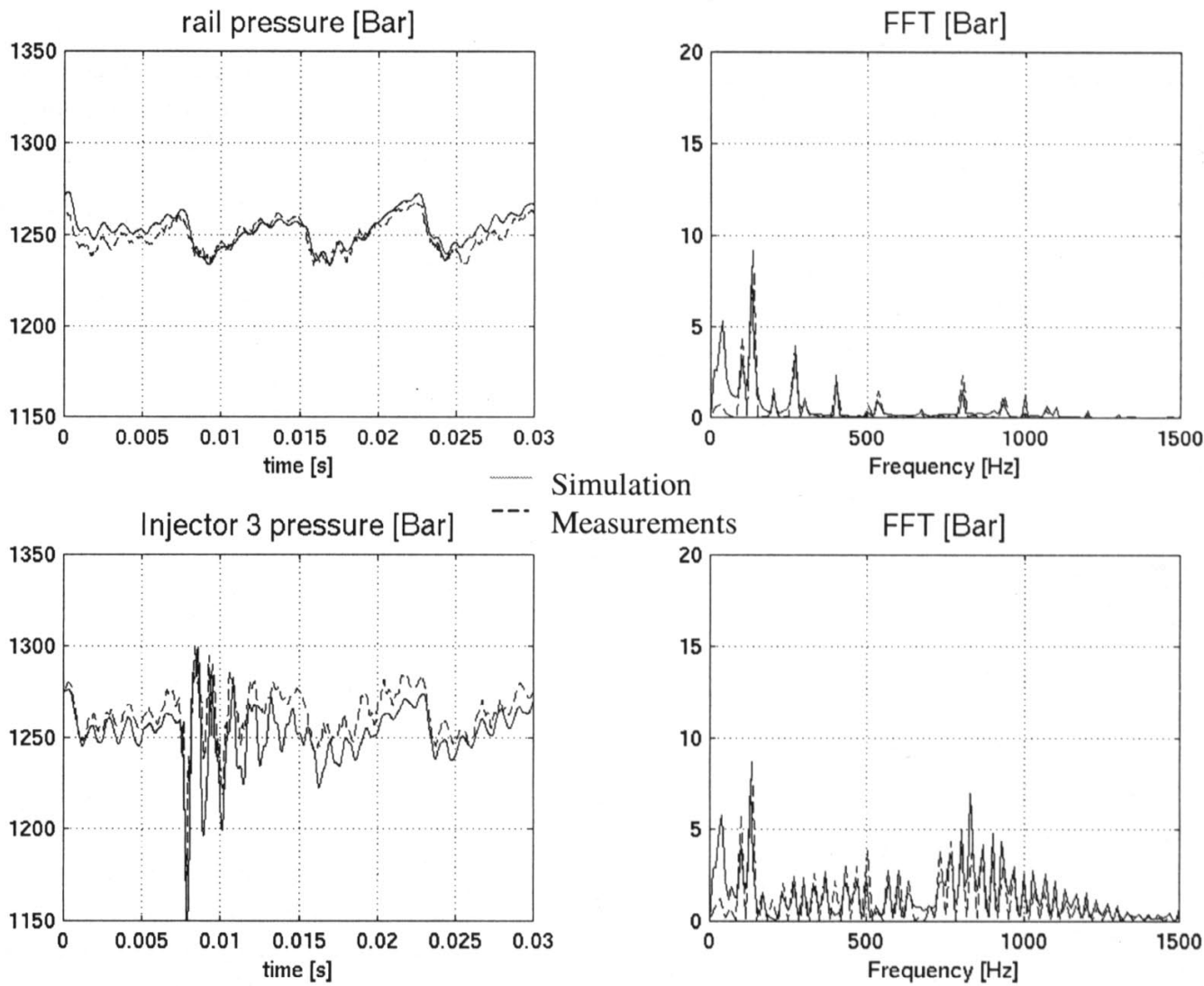

Simulation

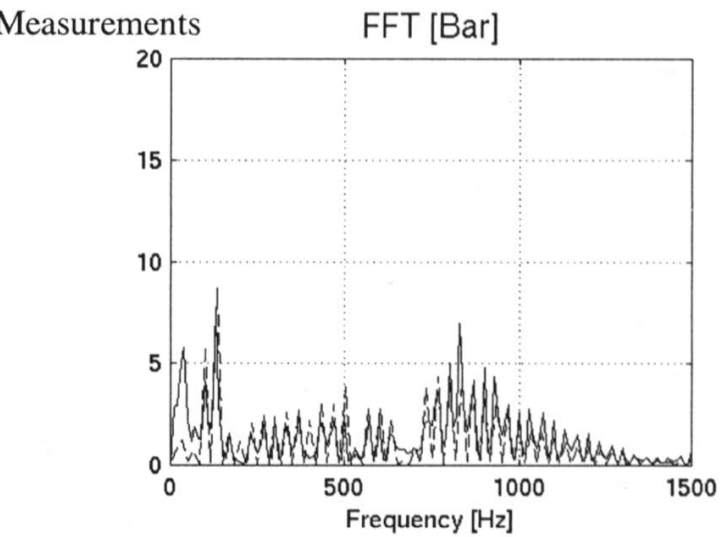

Figure 12 1-D simulation of the Common-Rail sytem 